\section{Consumo de cafeína durante a gestação e transtorno de déficit de atenção e hiperatividade (TDAH): uma revisão sistemática da literatura}

\author{
Caffeine consumption during pregnancy and \\ attention deficit hyperactivity disorder (ADHD): \\ a systematic literature review
}

\author{
Consumo de cafeína durante el embarazo y \\ trastorno por déficit de atención e hiperactividad \\ (TDAH): una revisión sistemática de la literatura
}

\author{
1 Universidade Federal de \\ Pelotas, Pelotas, Brasil. \\ 2 Universidade Federal do \\ Rio Grande, Rio Grande, \\ Brasil. \\ Correspondência \\ B. D. P. Silva \\ Universidade Federal de \\ Pelotas. \\ Rua Marechal Deodoro 1160 , \\ Pelotas, RS \\ 96020-220, Brasil. \\ bianca.delponte@gmail.com
}

\begin{abstract}
This aim of this study was to conduct a systematic literature review on the association between maternal caffeine intake during pregnancy and attention deficit hyperactivity disorder (ADHD) in childhood. The systematic multiple-stage literature search in PubMed, LILACS, BIREME, and PsycINFO was limited to research in human subjects and published in Portuguese, English, and Spanish. A total of 373 references were retrieved. Of these, only five met the study's objectives and were kept in the review. Most of the studies employed a longitudinal design, were conducted in developed countries, and were published in the last five years. Only one study found a positive association. Studies on caffeine consumption during pregnancy and ADHD are scarce, with conflicting results and several methodological difficulties such as lack of standardized outcome measures.
\end{abstract}

Caffeine; Pregnancy; Attention Deficit Disorder with Hyperactivity
Bianca Del Ponte da Silva 1

Luciana Anselmi 1,2

Vera Schmidt 1

Iná S. Santos 1

\section{Resumo}

O objetivo do presente estudo foi avaliar, mediante revisão sistemática da literatura, as evidências acerca da associação entre consumo materno de cafeína durante a gestação e transtorno de déficit de atenção e hiperatividade (TDAH) na infância. A busca na literatura ocorreu de forma sistemática, em múltiplas etapas, nas bases PubMed, LILACS, BIREME e PsycINFO, com limites para artigos publicados em português, inglês e espanhol, realizados em humanos. Foram encontradas 373 referências. Dessas, somente cinco foram mantidas, por atenderem ao objetivo deste estudo. Os cinco trabalhos foram realizados em países desenvolvidos; a maioria utilizou delineamento longitudinal e foi publicada nos últimos cinco anos. Apenas um estudo encontrou associação positiva. Estudos sobre o consumo de cafeína na gestação e TDAH são escassos, com resultados controversos e se deparam com várias dificuldades metodológicas, como falta de padronização na definição do desfecho.

Cafeína; Gravidez; Transtorno do Déficit de Atenção com Hiperatividade 


\section{Introdução}

O transtorno de déficit de atenção e hipetatividade (TDAH) é o mais prevalente transtorno neuropsiquiátrico em todo o mundo, afetando entre 5-10\% das crianças em idade escolar, e persistindo até a adolescência e a idade adulta em cerca de $30-50 \%$ desses 1,2 . O distúrbio é caracterizado por desatenção, hiperatividade e impulsividade e, para o diagnóstico, deve ter início antes dos 12 anos de idade ${ }^{3}$. Pode estar associado ao comprometimento em uma variedade de contextos na vida da criança, podendo levar, em casos de persistência na vida adulta, a significativas dificuldades acadêmicas, sociais, profissionais e interpessoais 4 .

A fisiopatologia do TDAH é complexa e parcialmente compreendida, sem que haja uma causa específica, sugerindo etiologia multifatorial, com uma combinação de fatores ambientais, genéticos e biológicos 1 . Fatores nutricionais no período gestacional, como ingestão de ácido fólico 5 , ferro 6 , ômega 37 e cafeína 8 têm sido investigados como determinantes de TDAH. Em animais, a exposição intrauterina a cafeína, em doses equivalentes a ingestão de 10-12 xícaras de café ao dia por humanos, foi associada com aumento da atividade motora, sugerindo a possibilidade de um efeito do consumo de cafeína durante a gestação sobre o déficit de atenção e a hiperatividade nos filhos 9,10.

A cafeína é um alcaloide do grupo das xantinas que exerce efeito sobre o sistema nervoso central 11. É comumente consumida no mundo todo, inclusive por gestantes. As principais fontes de cafeína são café, erva mate, chá preto (chá da Índia), cacau e bebidas à base de cola (refrigerantes como Coca-Cola, Pepsi e Guaraná), além de fazer parte da composição de muitos medicamentos, já que exerce efeitos farmacológicos, agindo como um antagonista de receptores de adenosina 12 .

Assim, o objetivo do presente estudo foi realizar uma revisão sistemática na literatura para identificar estudos em humanos que avaliaram a associação entre o consumo materno de cafeína durante a gestação e TDAH.

\section{Metodologia}

A busca na literatura com o objetivo de identificar artigos que avaliaram o consumo de cafeína durante a gestação como exposição e TDAH como desfecho foi realizada em junho de 2014. O processo ocorreu em múltiplas etapas, inicialmente com base na leitura dos títulos, em seguida pela leitura dos resumos e, por último, pela leitura completa dos artigos selecionados. A estratégia de busca ocorreu separadamente em cada uma das bases PubMed, LILACS, BIREME e PsycINFO.

A busca inicial foi feita na base de dados PubMed, com limites para artigos publicados em português, inglês e espanhol, realizados em humanos. Os termos utilizados foram Attention Deficit Hyperactivity Disorder (ADHD), Attention Deficit Hyperactivity (ADH), hyperactivity, mental health, behaviour, coffee, caffeine, gestation e pregnancy. Outras bases de dados foram rastreadas (BIREME, LILACS, e PsycINFO), utilizando os mesmos descritores. Além disso, foi realizada uma revisão nas referências dos artigos selecionados.

O processo de revisão foi realizado por dois revisores independentes, cujas discordâncias foram discutidas até o consenso. Como critérios de inclusão, os artigos deveriam avaliar o consumo de cafeína na gestação como exposição e o déficit de atenção/hiperatividade ou TDAH como desfecho. Cada artigo incluído na revisão foi avaliado quanto à adequação metodológica empregada na seleção da amostra, coleta e análise dos dados, de acordo com o recomendado na literatura 13. Os seguintes itens foram avaliados: representatividade da amostra, delineamento apropriado, presença de cegamento para avaliação de desfechos, realização de treinamento e padronização de medidas, utilização de instrumentos adequados para a avaliação da exposição e do desfecho, análise estatística apropriada, controle para fatores de confusão e cálculo de poder.

\section{Resultados}

A Figura 1 apresenta o fluxograma da seleção dos artigos. A estratégia de busca identificou 373 publicações na base PubMed e oito em outras bases de dados (BIREME, LILACS, e PsycINFO), mas três delas eram duplicatas. Após leitura dos títulos foram selecionados 20 registros. As 358 exclusões ocorreram porque os artigos não avaliavam o desfecho de interesse (124), nem a exposição de interesse (101) e outros eram artigos teóricos (133). As 20 publicações selecionadas foram importados para uma biblioteca do EndNote (Thomson Reuters. http://www.endnote.com/), na qual foi realizada a leitura dos resumos e seleção dos artigos para a leitura na íntegra. Nessa etapa, 13 artigos foram excluídos por não avaliarem o desfecho de interesse, restando sete para leitura na íntegra. Após a leitura na íntegra, dois artigos foram excluídos: um por não avaliar o consumo de cafeína; outro por não avaliar déficit de atenção/hiperatividade. Dessa forma, apenas 
Fluxograma da revisão de literatura sobre o consumo de cafeína durante a gestação e transtorno de déficit de atenção e hiperatividade (TDAH).

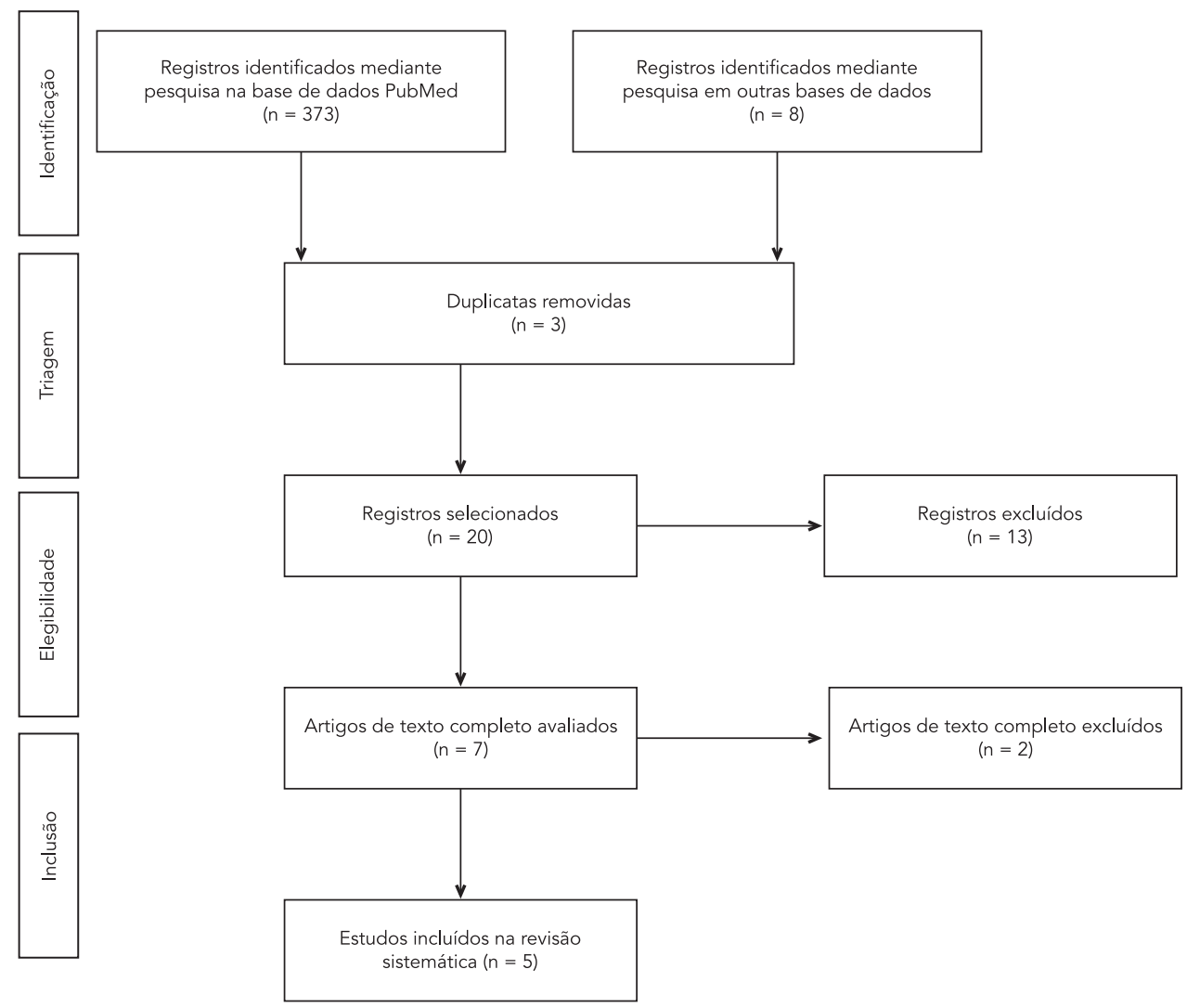

cinco publicações 8,14,15,16,17 atenderam aos critérios de inclusão para esta revisão.

Os cinco estudos foram realizados em países desenvolvidos e a maioria utilizou delineamento longitudinal. Exceto o de Barr \& Streissguth 14, publicado em 1991, os demais são estudos recentes, com publicação nos últimos cinco anos. Todos avaliaram desfechos de saúde mental em crianças com idades variando de 18 meses até 12 anos, e dois deles analisaram especificamente o TDAH, enquanto os outros mediram um conjunto de problemas de comportamento, incluindo TDAH.

Os instrumentos utilizados para avaliar o déficit de atenção/hiperatividade diferiram de acordo com o estudo, e a maioria empregou testes de rastreamento: Strengths and Difficulties Questionnaire (SDQ) 18, Child Behavior Checklist (CBCL) 19 e Continuous Performance Test (CPT) 20; apenas um utilizou avaliação clínica com base nos critérios diagnósticos da Classificação Internacional de Doenças, 10a revisão (CID-10) e do Manual Diagnóstico e Estatístico de Transtornos Mentais, 4a revisão (DSM-IV).

A exposição à cafeína foi identificada com base no consumo de alguns alimentos que apresentavam a substância em sua composição (café, bebidas à base de cola, chá preto e chocolate). Entre os estudos identificados, um examinou somente o consumo de café 17 , os demais investigaram o consumo de café, chá e cola, e dois deles 14,16 incluíram o consumo de chocolate. A quantidade de cafeína contida em cada tipo de bebida foi estimada por análises laboratoriais conduzidas pelos próprios estudos ou em parâmetros estabelecidos em estudos prévios. Com a informação sobre a concentração de cafeína nos alimentos e a porção consumida, foi possível obter a quantidade total de cafeína ingerida por dia, que era apresentada em miligramas. Na maioria dos 
estudos, a variável foi categorizada, e os limites das categorias diferiram entre as publicações.

A Tabela 1 apresenta um resumo das características metodológicas dos artigos selecionados para esta revisão, organizados por ordem cronológica, de acordo com a data de publicação. A primeira publicação foi a de Barr \& Streissguth 14 realizada com dados de um estudo longitudinal prospectivo, iniciado em 1974 nos Estados Unidos, com 1.529 gestantes, para investigar o efeito das exposições no pré-natal sobre a saúde da criança. Foram selecionadas gestantes de dois centros de saúde de Seattle, antes do 50 mês de gestação, momento em que elas foram entrevistadas na própria residência. O estudo avaliou a dieta das gestantes pelo Questionário de Frequência Alimentar (QFA), que estimava o consumo de cafeína pela ingestão de café, chocolate, chá e bebidas à base de cola. Para estimar a quantidade de cafeína presente em cada um dos alimentos, os autores realizaram análises laboratoriais, o que permitiu contabilizar a quantidade total em mg consumida por dia. Em 1991, Barr \& Streissguth ${ }^{14}$, utilizando dados desse estudo, avaliaram a associação entre o consumo de cafeína durante a gestação e o neurocomportamento dos filhos aos sete anos. Na análise, o consumo de cafeína foi avaliado em três categorias: $\leq 295$, 296-443 $\mathrm{e} \geq 444 \mathrm{mg} /$ dia. $\mathrm{O}$ estudo mostrou uma alta frequência de consumo de cafeína durante gestação $(99,4 \%)$. O chocolate foi a fonte de cafeína mais frequentemente consumida (83\%), seguido de chá preto $(81 \%)$, café $(69 \%)$ e bebidas à base de cola (53\%). A exposição foi detalhadamente mensurada, incluindo informações sobre as quantidades consumidas e utilização de diferentes fontes de cafeína. O desfecho foi avaliado pelo CPT, um instrumento de rastreamento que mede atenção e impulsividade, de acordo com duas dimensões (erro de omissão e erro de coomissão), sendo utilizado como auxiliar no processo diagnóstico de TDAH. Os resultados do estudo em tela mostraram que, após ajuste para características maternas (idade, escolaridade, dieta, uso de álcool e tabagismo durante a gestação) e da criança (sexo e ordem de nascimento), não houve associação entre o consumo de cafeína durante a gestação e a atenção $(p=0,925$ para associação com erro de omissão; e p = 0,861 para erro de coomissão).

Linnet et al. 16 realizaram um estudo longitudinal, na Dinamarca, que incluiu 24.068 crianças de 3 a 12 anos de idade, com o objetivo de avaliar a exposição intrauterina à cafeína e o risco de TDAH. As mães foram recrutadas e entrevistadas com 16 semanas de gestação. O consumo de cafeína foi aferido por questões sobre o número de xícaras de café, chá e bebidas à base de cho- colate ou cola consumidas diariamente. Além disso, foi realizada análise laboratorial para obter a concentração de cafeína contida em cada uma dessas substâncias, mas, sobre o consumo de café, os autores não especificaram o tipo (em pó, solúvel ou descafeinado), justificando ser baixo o consumo de café solúvel e descafeinado entre os dinamarqueses (Danish Coffee Information. http://www.kaffeinfo.dk; 2005). Não foram apresentadas informações sobre o tamanho das porções, sendo apenas descrito o consumo de copos ou xícaras. A avaliação do desfecho foi clínica, utilizando os critérios diagnósticos da CID10 e do DSM-IV. Em uma análise não ajustada, o consumo de mais de $1.000 \mathrm{mg}$ cafeína/dia esteve associado ao TDAH (RR = 3,0; IC95\%: 1,3-6,8). Mas, após ajuste para tabagismo, consumo de álcool, idade e escolaridade maternas, tipo de trabalho, tipo de habitação e história de hospitalização dos pais por problemas psiquiátricos, além do sexo da criança, a associação se desfez (RR = 2,3; IC95\%: 0,9-5,9). Foram realizadas análises complementares que excluíram crianças com histórico familiar de doenças psiquiátricas, com o objetivo de reduzir o efeito de fatores genéticos, mantendo-se os resultados anteriores inalterados.

O estudo de Chiu et al. 17 objetivou identificar fatores pré, peri e pós-natal, além da saúde mental materna, associados a desfechos de saúde mental e comportamento da criança. Foi um estudo transversal, de base populacional, conduzido em Taiwan, com uma amostra de 1.391 crianças de 4 a 9 anos de idade. As mães responderam a um questionário que investigava, retrospectivamente, informações sobre o consumo de alimentos no período gestacional. O consumo de cafeína foi aferido por uma pergunta sobre o consumo de pelo menos uma xícara de café por semana. Para as análises, utilizaram a variável na forma dicotômica (consumo semanal de café: sim ou não). Os problemas de comportamento nas crianças foram investigados com uso do CBCL, um instrumento de rastreio, que avalia a presença, entre outros, de problemas de atenção. O consumo de café não esteve associado aos problemas de atenção.

Bekkhus et al. 15, na Noruega, com uma população de 25.343 crianças de 18 meses, investigaram a associação entre a exposição intrauterina à cafeína na 17ạ e 30a semanas de gestação e sintomas de déficit de atenção e hiperatividade aos 18 meses, sugestivos de TDAH mais tarde. A cafeína foi investigada pelo consumo diário de café, chá preto e bebidas à base de cola, sendo consideradas diferentes porções (xícaras, canecas ou garrafas), que posteriormente foram transformados em uma porção padrão. A quantidade de cafeína 
Tabela 1

Descrição dos estudos que investigaram o consumo materno de cafeína durante a gestação e transtorno de déficit de atenção e hiperatividade (TDAH) incluídos na revisão sistemática da literatura.

\begin{tabular}{|c|c|c|c|c|c|c|c|}
\hline Estudo (país/ano) & Delineamento & $\begin{array}{l}\text { População/ } \\
\text { Idade }\end{array}$ & Desfecho & Instrumento & $\begin{array}{l}\text { Exposição } \\
\text { principal }\end{array}$ & Resultados & Limitações \\
\hline $\begin{array}{l}\text { Barr \& Streissguth } \\
14 \text { (Estados } \\
\text { Unidos/1991) }\end{array}$ & $\begin{array}{c}\text { Coorte } \\
\text { prospectiva }\end{array}$ & $\begin{array}{c}\mathrm{N}=500 ; \\
\text { crianças com } \\
7 \text { anos }\end{array}$ & $\begin{array}{l}\text { Neurocom- } \\
\text { portamento }\end{array}$ & CPT & $\begin{array}{l}\text { Consumo de } \\
\text { cafeína mg/ } \\
\text { dia (café, } \\
\text { chá, bebidas } \\
\text { contendo cola } \\
\text { e chocolate). } \\
\text { Coletado no } \\
\text { 5o mês de } \\
\text { gestação }\end{array}$ & $\begin{array}{c}\text { Não houve } \\
\text { associação entre } \\
\text { o consumo } \\
\text { de cafeína e } \\
\text { os resultados } \\
\text { neurocompor- } \\
\text { tamentais }\end{array}$ & $\begin{array}{l}\text { O artigo não } \\
\text { descreve as } \\
\text { perdas de } \\
\text { acompanha- } \\
\text { mento }\end{array}$ \\
\hline $\begin{array}{l}\text { Linnet et al. } 16 \\
\text { (Dinamarca/2009) }\end{array}$ & $\begin{array}{c}\text { Coorte } \\
\text { prospectiva }\end{array}$ & $\begin{array}{c}\mathrm{N}=24.068 \\
\text { crianças de } 3 \mathrm{a} \\
12 \text { anos }\end{array}$ & TDAH & CID-10, DSM-IV & $\begin{array}{l}\text { Consumo de } \\
\text { cafeína mg/ } \\
\text { dia (café, } \\
\text { chá, bebidas } \\
\text { contendo cola } \\
\text { e chocolate). } \\
\text { Coletado na } \\
\text { 16a semana de } \\
\text { gestação }\end{array}$ & $\begin{array}{c}\text { O consumo } \\
\text { de mais de } \\
1.000 \mathrm{mg} \\
\text { de cafeína/ } \\
\text { dia esteve } \\
\text { associado ao } \\
\text { TDAH (RR = 3,0; } \\
\text { IC95\%: 1,3-6,8), } \\
\text { após ajuste a } \\
\text { associação se } \\
\text { desfez (RR = 2,3; } \\
\text { IC95\%: 0,9-5,9) }\end{array}$ & - \\
\hline $\begin{array}{l}\text { Chiu et al. } 17 \\
\text { (China/2009) }\end{array}$ & Transversal & $\begin{array}{c}\mathrm{N}=1.391 \\
\text { crianças de } 4 \mathrm{a} \\
9 \text { anos }\end{array}$ & $\begin{array}{l}\text { Problemas de } \\
\text { comportamento }\end{array}$ & $\mathrm{CBCL}$ & $\begin{array}{l}\text { Consumo } \\
\text { de café na } \\
\text { gestação. } \\
\text { Coletado aos } \\
\text { 4-9 anos da } \\
\text { criança }\end{array}$ & $\begin{array}{c}\text { Não houve } \\
\text { associação entre } \\
\text { o consumo } \\
\text { de cafeína e a } \\
\text { atenção }\end{array}$ & $\begin{array}{l}\text { Exposição } \\
\text { obtida } \\
\text { retrospecti- } \\
\text { vamente com } \\
\text { frequência } \\
\text { de consumo } \\
\text { semanal sem } \\
\text { avaliar quanti- } \\
\text { dades }\end{array}$ \\
\hline $\begin{array}{l}\text { Bekkhus et al. } 15 \\
\text { (Noruega/2010) }\end{array}$ & $\begin{array}{c}\text { Coorte } \\
\text { prospectiva }\end{array}$ & $\begin{array}{c}\mathrm{N}=25.343 \\
\text { crianças com } \\
18 \text { meses }\end{array}$ & $\begin{array}{c}\text { Problemas } \\
\text { de atenção/ } \\
\text { hiperatividade }\end{array}$ & $\mathrm{CBCL}$ & $\begin{array}{l}\text { Consumo de } \\
\text { cafeína mg/ } \\
\text { dia (café, chá } \\
\text { e bebidas } \\
\text { contendo cola). } \\
\text { Coletado na 17ạ } \\
\text { e 30a semanas } \\
\text { de gestação }\end{array}$ & $\begin{array}{c}\text { Encontrou } \\
\text { associação entre } \\
\text { o consumo de } \\
\text { cafeína nas } \\
\text { primeiras } 17 \\
\text { semanas de } \\
\text { gestação e os } \\
\text { sintomas de } \\
\text { TDAH ( } p< \\
0,001 \text { ) }\end{array}$ & $\begin{array}{c}\text { O artigo não } \\
\text { descreve as } \\
\text { perdas } \\
\text { de acompanha- } \\
\text { mento }\end{array}$ \\
\hline $\begin{array}{l}\text { Loomans et al. } 8 \\
\text { (Holanda/ 2012) }\end{array}$ & $\begin{array}{c}\text { Coorte } \\
\text { prospectiva }\end{array}$ & $\begin{array}{c}\mathrm{N}=3.439 \\
\text { crianças de } \\
5-6 \text { anos }\end{array}$ & $\begin{array}{c}\text { Deficit de } \\
\text { atenção/ } \\
\text { hiperatividade }\end{array}$ & SDQ & $\begin{array}{l}\text { Consumo de } \\
\text { cafeína mg/dia } \\
\text { (café, bebidas } \\
\text { contendo cola e } \\
\text { chá). Coletado } \\
\text { na 16a semana } \\
\text { de gestação }\end{array}$ & $\begin{array}{c}\text { Não houve } \\
\text { associação entre } \\
\text { o consumo de } \\
\text { cafeína durante } \\
\text { a gestação e o } \\
\text { desfecho }\end{array}$ & - \\
\hline
\end{tabular}

CBCL: Child Behavior Checklist; CID-10: Classificação Internacional das Doenças, 10a revisão; CPT: Continuous Performance Test; DSM-IV: Manual Diagnóstico e Estatístico das Doenças Mentais; IC95\%: intervalo de 95\% de confiança; RR: risco relativo; SDQ: Strengths and Difficulties Questionnaire. 
contida em cada tipo de bebida foi estimada com base em parâmetros pré-estabelecidos, sendo gerada uma variável quantitativa (consumo total de cafeína em mg/dia). Os sintomas de déficit de atenção e hiperatividade foram analisados de forma combinada e separada, mediante três itens da subescala "excessivamente ativo" do CBCL 1.5-5, utilizada para rastreamento de problemas de comportamento em crianças de 1,5 a 5 anos. Os resultados mostraram associação entre o consumo de cafeína durante as primeiras 17 semanas de gestação e a presença de sintomas de TDAH $(\mathrm{p}<0,001)$, mesmo após ajuste para confundidores (características maternas: escolaridade, idade, situação conjugal, tabagismo e consumo de álcool na gestação; características da criança: peso ao nascer e circunferência cefálica). Já na análise da $30^{a}$ semana, após ajuste, o efeito não permaneceu significativo, no entanto, ao analisar déficit de atenção e hiperatividade separadamente, foi observado um efeito significativo sobre a hiperatividade $(\mathrm{p}<0,01)$. Foram, ainda, analisados os efeitos das diferentes fontes de cafeína (refrigerante, café e chá) separadamente. Apenas o refrigerante apresentou efeito sobre o déficit de atenção/hiperatividade $(\mathrm{p}<0,001)$.

Loomans et al. 8, utilizando dados de uma coorte de nascimentos de Amsterdã (Holanda), realizaram um estudo com 3.439 crianças de 5-6 anos de idade, para avaliar o efeito da cafeína no período pré-natal sobre o comportamento na infância. O consumo de cafeína foi aferido durante a gestação, mais precisamente nas primeiras 16 semanas, por questionários com perguntas sobre a ingestão de fontes de cafeína como café, chá e cola, em porções diárias. O total de cafeína ingerido foi estimado com base no Dutch Food Composition Database, que fornece informações sobre a composição nutricional e conteúdo de cafeína dos alimentos. O total de cafeína consumido em miligramas foi categorizado em 0-85, 86-255, 256-425 e > 425mg/dia. Os desfechos foram avaliados pelo SDQ, um instrumento de rastreamento que consiste em 25 itens, que avaliam mediante cinco subescalas vários aspectos do comportamento, entre eles: problemas de relacionamento, de conduta, emocional, pró-social ou problemas de atenção e hiperatividade. Em uma análise ajustada para características maternas (idade, cor da pele, escolaridade, situação conjugal, tabagismo, alcoolismo e ansiedade), tamanho da família e sexo da criança, não foi encontrado efeito da cafeína sobre o risco de TDAH nas crianças, porém os filhos de mães na categoria de maior consumo ( $>425 \mathrm{mg} /$ dia) apresentaram OR = 1,08 $(0,55-2,12)$ em comparação aos de mães da categoria de menor consumo $(0-85 \mathrm{mg} /$ dia). Após ajuste para os possíveis mediadores peso ao nascer e idade gestacional, o resultado permaneceu igual.

\section{Discussão}

Esta revisão sistemática identificou apenas cinco estudos que avaliaram os efeitos do consumo materno de cafeína durante a gestação e a ocorrência de TDAH na infância, sendo seus achados controversos. Dentre os quatro estudos mais bem delineados e analisados 8,14,15,16, apenas um encontrou associação 15 entre consumo de cafeína e hiperatividade aos 18 meses de idade. Assim, a evidência atualmente disponível é insuficiente para que se possa afirmar ou refutar a associação entre o prevalente hábito de consumir alimentos ricos em cafeína na gestação e a ocorrência de TDAH entre os filhos.

A capacidade de um estudo identificar a potencial associação entre uma exposição e um desfecho depende, dentre outros fatores, da população em estudo, do tamanho e de como a amostra é selecionada, do modo como a exposição e o desfecho são aferidos, além do adequado controle para fatores de confusão. Entre os estudos que não encontraram associação entre consumo materno de cafeína e TDAH, algumas vantagens e limitações devem ser destacadas. Os estudos de Barr \& Streissguth 14, Linnet et al. 16, Loomans et al. 8 e Bekkhus et al. 15 foram metodologicamente muito bem conduzidos. A exposição foi detalhadamente mensurada, incluindo informações sobre as quantidades consumidas e utilizando diferentes fontes de cafeína. Todos avaliaram a quantidade de cafeína contida em cada tipo de bebida, a qual foi estimada com base em análises laboratoriais conduzidas pelos próprios estudos ou em parâmetros estabelecidos por estudos prévios. Ademais, a maioria analisou a variável em mais de duas categorias, permitindo a avaliação da dose-resposta. Contudo, as quantidades foram estimadas com base em medidas caseiras, incorrendo em possível falta de precisão na estimativa da dose consumida. Não houve distinção entre o tipo de café, se solúvel ou em pó, um limitante, tendo em vista a diferença na concentração de cafeína entre eles 21 . Tais estudos utilizaram delineamento longitudinal prospectivo, ideal para investigar associação entre exposições precoces e desfechos na infância, pois diminui a chance de viés de memória. Além disso, realizaram controle para diversos fatores de confusão. $\mathrm{O}$ estudo de Linnet et al. ${ }^{16}$, que incluiu um grande número de crianças (24.068), empregou um critério mais exigente do que o empregado pelos demais autores (CID-10 e DSM-IV contra testes de rastreamento). 
Já a descrição do estudo transversal de Chiu et al. 17 apresentou algumas limitações. Primeiro, não foram apresentados o cálculo de tamanho de amostra nem o processo de amostragem. No que diz respeito à exposição, apenas o café foi investigado como fonte de cafeína. Ainda, a informação sobre o consumo de cafeína foi obtida retrospectivamente, abrangendo um período longo de tempo (4 a 9 anos), o que pode ter acarretado viés de memória. Além do mais, a frequência de consumo estimada foi semanal, colocando na mesma categoria de consumo mães que consumiam café todos os dias ou em mais de um dia na semana, com as que consumiam apenas uma vez por semana. E, por último, a falta de informação sobre quantidades consumidas impossibilitou análises de efeito dose-resposta.

O estudo de Bekkhus et al. 15 foi o único que encontrou associação entre o consumo de cafeína e o TDAH. Porém, para interpretação correta desses resultados, algumas limitações devem ser consideradas. A associação foi verificada apenas com hiperatividade e não com déficit de atenção. Como a história natural do TDAH não é totalmente conhecida, é possível que as crianças estudadas fossem muito jovens para apresentarem os sintomas específicos de hiperatividade ou déficit de atenção. Além disso, para o diagnóstico de TDAH é necessário que a criança apresente os sintomas em pelo menos dois ambientes (domiciliar e escolar), uma vez que as crianças tendem a se comportar diferentemente no ambiente domiciliar e na escola 22 , e neste estudo foi considerado apenas o ambiente domiciliar, o que aumenta a possibilidade de resultados falsopositivos, já que as mães tendem a superestimar os problemas de comportamento dos filhos 23 . O estudo envolveu um número grande de crianças (mais de 25 mil), fato que aumenta o poder de detectar como significativas pequenas diferenças entre os grupos analisados, especialmente porque a definição operacional do desfecho baseouse em um teste de rastreamento.

Os critérios adotados e as medidas utilizadas para o diagnóstico do TDAH desempenham um papel fundamental nos resultados gerados 24 . Instrumentos de rastreamento possuem maior sensibilidade e menor especificidade, tendendo a superestimar as prevalências dos desfechos de saúde. Nos estudos incluídos nesta revisão, o déficit de atenção/hiperatividade foi avaliado de acordo com diferentes escalas de triagem (CPT, SDQ e CBCL) e os critérios diagnósticos da CID10 e do DSM-IV, dificultando a comparabilidade entre os achados.

Em estudos que envolvem consumo alimentar importa atentar para a complexidade da dieta humana 25 . A dieta é composta por uma varieda- de de substâncias (nutrientes essenciais: vitaminas, minerais, lipídeos e aminoácidos; fontes de energia: carboidrato, proteína, lipídeos e álcool; toxinas; enzimas; e compostos inorgânicos), que interagem entre si, agindo antagônica ou sinergicamente na absorção dos mesmos. Não só em razão da complexidade, torna-se difícil a obtenção de dados precisos sobre dieta, visto que os indivíduos não recordam com exatidão os alimentos consumidos, mesmo que recentemente. Além disso, não são capazes de lembrar com precisão os momentos em que ocorreram alterações nos hábitos alimentares 25 . Apesar da dificuldade em avaliar o consumo alimentar, essa investigação é imprescindível à epidemiologia nutricional.

Tal dificuldade está presente na mensuração e quantificação do consumo de cafeína. A cafeína é encontrada em diversas fontes, sendo a principal o café. Dessa forma, a mensuração precisa do seu consumo é de extrema importância para a quantificação da ingestão de cafeína. Esse detalhamento envolve, sobretudo, forma de preparo, diluição, tipo de café e porções consumidas. Camargo \& Toledo 21 , avaliando a quantidade de cafeína nos diferentes tipos de café normalmente consumidos pela população brasileira, observaram grande variação no teor da substância, conforme a quantidade de pó utilizada, o tipo de café e a forma de preparo. Bracken et al. 26 observaram variação até quando a mesma pessoa fervia o café ou o chá em condições aparentemente idênticas e no mesmo dia.

Estudos com animais mostraram efeito positivo da ingestão de cafeína sobre a atividade motora 9,10. Porém, a quantidade de cafeína administradas nestes experimentos foram superiores ao correspondente a 10 xícaras/dia, quantidade superior à habitualmente consumida por humanos. A maioria dos estudos que investigaram em humanos o mesmo efeito, não encontrou associação. Entretanto, esses resultados podem ser devidos a limitações metodológicas, como as já apontadas.

Em resumo, são escassos os estudos que avaliaram o efeito do consumo de cafeína na gestação sobre o TDAH, o que não permite confirmar ou refutar o risco que a exposição oferece ao desenvolvimento desta morbidade na infância. Considerando que o TDAH é um dos transtornos mentais mais prevalentes entre as crianças, inclusive entre as brasileiras 27 , sendo a principal causa pela procura por atendimento aos serviços de saúde mental para crianças e adolescentes em países desenvolvidos 4; que é responsável por uma série de prejuízos, estando associado ao comprometimento em uma variedade de contextos na vida da criança, podendo levar a significativas dificuldades acadêmicas, familiares e 
sociais e, em casos de persistência na vida adulta, a problemas profissionais e interpessoais 4; e que é entendido como um transtorno crônico, que frequentemente persiste até a vida adulta, provocando prejuízos cumulativos 4 , mostra-se de suma importância identificar o risco associado ao consumo de cafeína, tendo em vista a possibilidade de realização de medidas preventivas aplicáveis ainda na vida intrauterina. A atual revisão da literatura mostrou que a resposta a esta importante pergunta permanece em aberto. Para melhorar a comparabilidade dos futuros estudos, recomenda-se que o instrumento de avaliação do desfecho seja padronizado. Também, com base na atual revisão, sugere-se que o ajuste para saúde mental materna, uso materno de substâncias durante a gestação e acompanhamento prénatal seja realizado em estudos que abordem esta importante questão de pesquisa.

\section{Resumen}

El objetivo de este estudio fue evaluar, a través de una revisión sistemática de la literatura, evidencias sobre la asociación entre el consumo de cafeína durante el embarazo y el trastorno por déficit de atención e hiperactividad (TDAH) en la infancia. Se realizó una búsqueda sistemática en la literatura, por etapas múltiples, en PubMed, LILACS BIREME y PsycINFO, limitándose a artículos publicados en portugués, inglés y español, realizados en estudios sobre humanos. Fueron localizadas 373 referencias. De ellas, apenas se mantuvieron cinco, por cumplir el objetivo de este estudio. Los estudios se realizaron en países desarrollados; el diseño longitudinal fue el más utilizado y se trata de publicaciones de los últimos cinco años. Sólo un estudio encontró asociación positiva. Los estudios sobre el consumo de cafeína durante el embarazo y el TDAH son escasos, con resultados controvertidos, y enfrentan varias dificultades metodológicas, como la no estandarización de la evaluación del resultado.

Cafeína; Embarazo; Trastorno por Déficit de Atención con Hiperactividad

\section{Colaboradores}

B. D. P. Silva e I. S. Santos participaram da concepção e projeto, análise e interpretação dos dados; participaram na redação do artigo e revisão crítica relevante do conteúdo intelectual, além da aprovação final da versão a ser publicada. Possuem responsabilidade por todos os aspectos do trabalho. L. Anselmi e V. Schmidt realizaram análise e interpretação dos dados; participaram na redação do artigo e revisão crítica relevante do conteúdo intelectual, além da aprovação final da versão a ser publicada. Possuem responsabilidade por todos os aspectos do trabalho.

\section{Agradecimentos}

Este artigo foi realizado com dados do estudo Coorte de Nascimentos de Pelotas, 2004, conduzido pelo Programa de Pós-graduação em Epidemiologia da Universidade Federal de Pelotas, com o apoio da Associação Brasileira de Saúde Coletiva (ABRASCO). O estudo foi financiado pelo Wellcome Trust, Organização Mundial da Saúde, Programa de Apoio a Núcleos de Excelência (PRONEX), Conselho Nacional de Desenvolvimento Científico e Tecnológico (CNPq), Ministério da Saúde e Pastoral da Criança. 


\section{Referências}

1. Biederman J. Attention-deficit/hyperactivity disorder: a selective overview. Biol Psychiatry 2005; 57:1215-20.

2. Clinical practice guideline: diagnosis and evaluation of the child with attention-deficit/hyperactivity disorder. American Academy of Pediatrics. Pediatrics 2000; 105:1158-70.

3. Araújo AC, Lotufo Neto F. A nova classificação americana para os transtornos mentais: o DSM-5. Rev Bras Ter Comport Cogn 2014; XVI:67-82.

4. Barkley RA. Transtorno de déficit de atenção/hiperatividade: manual para diagnóstico e tratamento. Porto Alegre: Artmed; 2008.

5. Scholl TO, Johnson WG. Folic acid: influence on the outcome of pregnancy. Am J Clin Nutr 2000; 71:1295-303.

6. Parsons AG, Zhou SJ, Spurrier NJ, Makrides M. Effect of iron supplementation during pregnancy on the behaviour of children at early school age: longterm follow-up of a randomised controlled trial. $\mathrm{Br}$ J Nutr 2008; 99:1133-9.

7. Hibbeln JR, Davis JM, Steer C, Emmett P, Rogers I, Williams C, et al. Maternal seafood consumption in pregnancy and neurodevelopmental outcomes in childhood (ALSPAC study): an observational cohort study. Lancet 2007; 369:578-85.

8. Loomans EM, Hofland L, van der Stelt O, van der Wal MF, Koot HM, van den Bergh BR, et al. Caffeine intake during pregnancy and risk of problem behavior in 5- to 6-year-old children. Pediatrics 2012; 130:e305-13.

9. Hughes RN, Beveridge IJ. Sex-and age-dependent effects of prenatal exposure to caffeine on openfield behavior, emergence latency and adrenal weights in rats. Life Sci 1990; 47:2075-88.

10. Nakamoto T, Roy G, Gottschalk SB, Yazdani M, Rossowska M. Lasting effects of early chronic caffeine feeding on rats' behavior and brain in later life. Physiol Behav 1991; 49:721-7.

11. Nehlig A. Pharmacological properties and neurophysiological effects of caffeine. Pharmacopsychoecologia 2002; 15:35-70.

12. International Agency for Research on Cancer. Coffee, tea, maté, methylxanthines and methylglyoxal. Lyon: International Agency for Research on Cancer; 1991. (Monographs on the Evaluation of Carcinogenic Risks to Humans, 51).

13. Rothman KJ, Greenland SG, Lash TL. Modern epidemiology. 3rd Ed. Philadelphia: Lippincott, Williams \& Wilkins; 2008.

14. Barr HM, Streissguth AP. Caffeine use during pregnancy and child outcome: a 7-year prospective study. Neurotoxicol Teratol 1991; 13:441-8.
15. Bekkhus M, Skjothaug T, Nordhagen R, Borge AI. Intrauterine exposure to caffeine and inattention/overactivity in children. Acta Paediatr 2010; 99:925-8.

16. Linnet KM, Wisborg K, Secher NJ, Thomsen PH, Obel C, Dalsgaard S, et al. Coffee consumption during pregnancy and the risk of hyperkinetic disorder and ADHD: a prospective cohort study. Acta Paediatr 2009; 98:173-9.

17. Chiu YN, Gau SS, Tsai WC, Soong WT, Shang CY. Demographic and perinatal factors for behavioral problems among children aged 4-9 in Taiwan. Psychiatry Clin Neurosci 2009; 63:569-76.

18. van Widenfelt BM, Goedhart AW, Treffers PDA, Goodman R. Dutch version of the Strengths and Difficulties Questionnaire (SDQ). Eur Child Adolesc Psychiatry 2003; 12:281-9.

19. Achenbach TM. Manual for the Child Behavior Checklist/6 and 182001 profile. Burling: University of Vermont; 2001.

20. Beck LH, Bransome Jr. ED, Mirsky AF, Rosvold HE, Sarason I. A continuous performance test of brain damage. J Consult Psychol 1956; 20:343-50.

21. Camargo MCR, Toledo MCF. Teor de cafeína em cafés brasileiros. Ciênc Tecnol Aliment 1998; 18:421-4.

22. Najman JM, Williams GM, Nikles J, Spence S, Bor W, O'Callaghan M, et al. Mothers' mental illness and child behavior problems: cause-effect association or observation bias? J Am Acad Child Adolesc Psychiatry 2000; 39:592-602.

23. Kroes G, Veerman JW, De Bruyn EEJ. Bias in parental reports? Maternal psychopathology and the reporting of problem behavior in clinic-referred children. Eur J Psychol Assess 2003; 19:195-203.

24. Offord DR. Child psychiatric disorders:prevalence and perpectives. Psychiatr Clin North Am 1985; 8:637-52.

25. Willett W, Sampson L. Foods and nutrients. In: Willett W, editor. Nutritional epidemiology. 3rd Ed Oxford: Oxford University Press; 2013. p. 17-33

26. Bracken MB, Triche EW, Grosso L, Hellenbrand K, Belanger K, Leaderer BP. Heterogeneity in assessing self-reports of caffeine exposure: implications for studies of health effects. Epidemiology 2002; 13:165-71.

27. Petresco S, Anselmi L, Santos IS, Barros AJ, Fleitlich-Bilyk B, Barros FC, et al. Prevalence and comorbidity of psychiatric disorders among 6-year-old children: 2004 Pelotas Birth Cohort. Soc Psychiatry Psychiatr Epidemiol 2014; 49:975-83.

Recebido em 19/Set/2014

Versão final reapresentada em 09/Jan/2015

Aprovado em 21/Jan/2015 